\title{
Einleitung: Komplementärmedizin als Chance
}

\author{
Hans-WolfGang Hoefert, ANdReas MichaLsen und BERnHARd UEHLEKE
}

Die Popularität von komplementär-alternativen und naturheilkundlichen Heilmethoden hat in den letzten Jahrzehnten kontinuierlich zugenommen, und dies ungeachtet der nicht immer vorliegenden Evidenz solcher Methoden. In der Krankenhauslandschaft ist die komplementär-alternative Medizin und die Naturheilkunde Medizin bisher in Form spezieller und unterschiedlichster Einrichtungen, teilweise durch weltanschaulich oder konfessionell geprägte Krankenhäuser sowie Spezialeinrichtungen für einzelne Therapierichtungen oder Erkrankungen vertreten. Der alternativ-komplementärmedizinische Bereich richtet sich unbedingt nach Standards und Leitlinien, orientiert sich allerdings an eigenen Qualitätskriterien. Krankenhäuser der Regelversorgung stehen daher vor der Frage, ob und inwieweit sie sich diesem Trend anschließen sollen, um damit auch den Erwartungen vieler Patienten entsprechen zu können. Jüngste Konzepte der Integrativen Medizin lassen erkennen, dass eine verstärkte zukünftige Implementierung solcher Heilmethoden in die Krankenhausmedizin bevorsteht.

\section{Konventionelle versus alternativ-komplementäre Medizin}

In der Öffentlichkeit werden die Begriffe „Alternativmedizin“ und „Komplementärmedizin“ teilweise synonym verwendet. Genau genommen umfasst „Alternativmedizin“ alle Methoden, die nicht in der konventionellen Medizin vertreten sind, von jener abgelehnt werden oder damit in Konkurrenz stehen, während „Komplementärmedizin“ sich auf solche Methoden bezieht, die von der konventionellen Medizin durchaus anerkannt sind und ergänzend zu konventionellen Verfahren eingesetzt werden. Insofern wurde der Begriff der „Alternativmedizin“ von den Fachkreisen weitgehend verlassen, da er nicht die Praxis abbildet, die 
heute in der Regel eine Kombination der besten Methoden beider Bereiche beinhaltet („Integrative Medizin“). Die Misteltherapie der anthroposophischen Medizin bei der Krebsbehandlung versteht sich heute kaum noch als Alternative zu einer chemotherapeutischen Behandlung, sondern als Ergänzung. Verfahren wie Yoga oder bestimmte Ernährungsformen fungieren hingegen nicht mehr als Alternativen oder Ergänzungen, sondern sind durch ihre starke Evidenz zunehmend auch assimilierte Teile der konventionellen Medizin. Zusammenfassend wird heute Alternativ- und Komplementärmedizin betrachtet als eine Vielzahl von therapeutischen Methoden, die der Prävention, Kuration und Rehabilitation dienen und je nach Gesundheitssystem mehr oder weniger als ergänzende Form zur konventionellen Medizin anerkannt werden (vgl. Falkenberg et al. 2012).

Dennoch bleibt es für viele Patienten ein Ziel, durch ein Verfahren der Naturheilkunde oder Komplementärmedizin schulmedizinische Medikamente einzusparen oder im Verlauf einer Therapie ggf. gar nicht mehr einnehmen zu müssen. Beispiele hierfür sind die Schmerzlinderung durch Akupunktur oder Blutdrucksenkung durch Aderlass, die damit sowohl ergänzend als auch zumindest in manchen Fällen substitutiv angewendet werden.

Aus medizinhistorischer Sicht kann sich der Status des einen oder anderen Verfahrens damit im Laufe der Zeit mehrfach wandeln. Viele Verfahren, die heute zum festen Bestand der konventionellen Medizin gerechnet werden, waren anfangs sogenannte Außenseitermethoden (z.B. bestimmte Verfahren der physikalischen Therapie), andere sind mangels Bewährung ausgesondert worden (Jütte 1996). So definiert denn auch das National Center for Complementary and Alternative Medicine (NCCAM) die Komplementärmedizin als „Gruppe verschiedener medizinischer und gesundheitlicher Systeme, Praktiken und Produkte, die gegenwärtig nicht als Teil der konventionellen Medizin angesehen werden“ (NCCAM 2008).

Es ist schwierig, eine klare Trennlinie zwischen konventioneller und alternativer bzw. komplementärer Medizin zu ziehen, wenn man sich allein nach den Kriterien der evidenzbasierten Medizin richtet. Hier zeigt sich, dass zwar die Methoden der Alternativ- und Komplementärmedizin in unterschiedlichem Maße solchen Kriterien genügen (Stiftung Warentest 2006, Hoefert \& Uehleke 2009, Singh \& Ernst 2009), dass dies aber auch für verschiedene konventionelle Verfahren gilt. Zudem wird besonders von Seiten der Homöopathie und der Anthroposophischen Medizin die Angemessenheit evidenzbasierter, d.h. auf dem Vergleich klinischer Gruppen beruhender Forschungsergebnisse für die Behandlung von Einzelfällen bezweifelt (so z.B. Kiene 2001). Auch die Frage, inwieweit die eine oder andere Methode auf Placebo-Effekten beruht, erscheint wenig weiterführend, weil solche Effekte selbst bei - nach evidenzbasierten Kriterien - bewährten Verfahren oder Medikamenten nicht auszuschließen sind und für alle Verfahren der Medizin einschließlich der Chirurgie zutreffen (vgl. Hoefert \& Uehleke 2009, 36ff.).

Angesichts der Schwierigkeiten, konventionelle, alternative und komplementäre Medizin praktisch und theoretisch voneinander abzugrenzen, bleiben - von we- 
nigen Ausnahmen abgesehen, die größtenteils der Esoterik zugerechnet werden können - vor allem pragmatische Erfahrungswerte, nach denen das eine oder andere Verfahren als hilfreich und angemessen gelten kann. Dies ist vermutlich der Hauptgrund, warum man es im englischsprachigen Raum bei Sammelbezeichnungen wie „Complementary and Alternative Medicine“ (CAM) für alle Verfahren belässt, die ausdrücklich nicht zum Kanon der konventionellen Medizin, d.h. zu den an den Universitäten gelehrten medizinischen Fächern gezählt werden bzw. in den offiziellen Diagnosesystemen ICD und DSM nicht erfasst werden. Ob ein Verfahren als alternativ (zu einem konventionellen Verfahren) gilt, hängt von dessen Verwendungsweise ab; in diesem Sinne ist zunächst jedes Verfahren als alternativ zu bezeichnen, das - aufgrund von noch fehlenden Evidenznachweisen - noch keinen Eingang in die offiziellen Klassifikationssysteme gefunden hat. Verfahren, die eine sinnvolle Ergänzung zu konventionellen Verfahren darstellen (weil sie die Wirkung eines konventionellen Verfahrens verstärken oder dessen Nebenwirkungen mildern), werden als komplementär bezeichnet; in diesem Sinne kommen auch Verfahren der konventionellen Medizin und nicht nur KMVerfahren im Hinblick auf den jeweiligen Verwendungszweck in Betracht.

Aus pragmatischen Gründen werden auch deshalb in Arztpraxen ebenso wie in Krankenhäusern einzelne Verfahren der Komplementärmedizin (KM) gebündelt oder ergänzend zu konventionellen Verfahren verwendet, ohne dass ein gemeinsamer theoretischer Hintergrund erkennbar wäre. Die Auswahl und Kombination erfolgt vielmehr eklektizistisch und richtet sich bestenfalls nach der internen Verträglichkeit (Ausschließung von negativen Wechselwirkungen). Die jeweiligen Kombinationen werden dann in Kombination mit der Schulmedizin und, soweit ein gewisse Evidenz vorliegt als „integrativ“ bezeichnet, während „ganzheitlich“ vor allem die Integration bio-psychosozialer, empathischer und psychosomatischer Anteile im Kontext eines Gesamtbehandlungsprogramms bezeichnet.

Da solche Kombinationen aber je nach Anbieter von Gesundheitsleistungen wechseln, gibt es auch nicht eine spezielle „integrative“ oder „ganzheitliche“ Medizin. Ebenso wenig gibt es die Traditionelle Chinesische Medizin (TCM), weil auch diese eine willkürliche Kombination von ausgewählten Verfahren darstellt, die sich in der chinesischen Medizin im Laufe der Jahrhunderte mehr oder weniger bewährt haben. Theoretisch fundiert und mit einem eingegrenzten Methodenarsenal versehen sind dagegen die „klassische“ Naturheilkunde, die Homöopathie, Ayurveda und die Anthroposophische Medizin, - was allerdings über die Bewährung dieser Methoden noch keine Aussagen zulässt.

\section{Charakteristika der KM-Nutzung}

Während der „erste“ Gesundheitsmarkt definiert ist durch klassische Leistungsanbieter bzw. Leistungsangebote wie stationäre und nicht-stationäre Dienstleistungen, Medizintechnik oder pharmazeutische Erzeugnisse, umfasst der „zweite“ Gesundheitsmarkt neben Dienstleistungen für Sport, Fitness, funktionelle Lebensmittel auch sonstige Dienstleistungen, die der komplementär-alterna- 
tiven Medizin zugerechnet werden können (Kartte \& Neumann 2008). Diesem „zweiten“ Markt wird in führenden Wirtschaftsgutachten (Deloitte 2010) ein hohes Wachstumspotenzial zugeschrieben.

\section{Prävalenzen}

Die Popularität von Methoden und Produkten der komplementär-alternativen Medizin in der Bevölkerung ist in den letzten Jahrzehnten noch weiter gewachsen. Bei den KM-Nutzern handelt es sich überwiegend um Frauen, um Personen mittleren Alters, und um solche mit gehobenem Bildungs- sowie Einkommensniveau (Ernst 200o). Diese international gültige Feststellung ist auch in verschiedenen Untersuchungen mit deutschsprachigen Nutzern bestätigt worden (GBE 2002, Wapf \& Busato 2007). Demnach nimmt die Tendenz zur KM-Nutzung mit steigender Schulbildung und höherem Haushaltseinkommen zu.

Nach derzeitigem Stand (Frass et al. 2012) schwanken die Prävalenzraten für die deutsch- und englischsprachigen Länder je nach Studie zwischen 5 und 75\% (Median $32,2 \%$ ). Im internationalen Vergleich erfahren in den deutschsprachigen Ländern Homöopathie und Akupunktur den größten Zuspruch (wobei fälschlicherweise unter Homöopathie auch Phytotherapie subsummiert wird und erstattungsfähige physikalische Therapien eher der konventionellen Medizin zugeordnet werden), in den englischsprachigen Ländern Chiropraktik, Phytotherapie, Massage und Homöopathie (wenn man „Beten“ ausklammert). Die Hauptindikationen sind Rückenschmerzen, Depressionen, Schlafstörungen, schwere Kopfschmerzen oder Migräne sowie Magen-Darm-Störungen.

\section{Präferenzen}

Generell wird die KM nach einer älteren deutschen Studie vor allem dann in Anspruch genommen, wenn bei akuten oder chronischen Leiden die erwarteten Heilerfolge der konventionellen Medizin ausbleiben, wenn die Nebenwirkungen konventioneller Medikamente gefürchtet werden (KM gilt als „natürlich“, „sanft“ und „nebenwirkungsfrei“) und wenn eine konventionelle (medikamentöse) Behandlung per Selbstmedikation unterstützt werden soll. KM wird aber nicht nur als „Begleitmedikation“ benutzt, sondern auch häufig als „Vorbeugemedikation“. Dazu kommt schließlich als weiteres Motiv für den Gebrauch von KM die bei manchen KM-Methoden erfahrene Kommunikation und Zuwendung, welche oft auch als „niederschwellige Psychotherapie“ bezeichnet wird (GBE 2002, 22ff.).

Fragt man nach der Präferenz für bestimmte KM-Methoden, dann fallen im internationalen Vergleich einige Unterschiede und Trends auf. In den USA erfreut sich beispielsweise Beten für die eigene Gesundheit nach den Untersuchungen von 1998 und 2004 größter Beliebtheit, eine Methode, die in europäischen Untersuchungen kaum eine Bedeutung hat (genauere Prozentangaben bei Hoefert \& Uehleke 2009) bzw. nicht zur Medizin gezählt wird. Im zeitlichen Vergleich zeigt sich in den USA ein Nachlassen der Popularität von Chiropraktik und Osteopa- 
thie, ein Zuwachs der Präferenz für Akupunktur, sowie eine konstant hohe Popularität für Phytotherapie und Entspannungsmethoden, Yoga und Atemtherapien. Die folgende Übersicht (s. Tab.1) gibt die ersten drei Rangplätze in nationalen Erhebungen wieder. Dabei ist $\mathrm{zu}$ berücksichtigen, dass die deutsche Untersuchung von Härtel \& Volger (2004) sich vorwiegend auf Naturheilverfahren konzentrierte und bei der ebenfalls deutschen Untersuchung von Bücker at al. (2008) nicht nach Nahrungsergänzungsmitteln und Vitaminen, die in den USA und Kanada recht beliebt sind, sowie nach Chiropraktik und Massagemethoden gefragt wurde, weil diese nach Meinung der Autoren der Konventionellen Medizin zuzurechnen seien.

Tab. 1 Popularität von KM-Methoden

\begin{tabular}{lllll}
\hline Eisenberg et al. 1998 & Barnes et al. 2004 & Härtel \& Volger 2004 & $\begin{array}{l}\text { Bücker et al. } \\
2008\end{array}$ & $\begin{array}{l}\text { Büssing et al. } \\
2011\end{array}$ \\
\hline $\begin{array}{l}\text { Selbst beten für die } \\
\text { eigene Gesundheit }\end{array}$ & $\begin{array}{l}\text { Selbst beten für } \\
\text { die eigene Ge- } \\
\text { sundheit }\end{array}$ & Bewegungstherapien & Akupunktur & Akupunktur \\
\hline Entspannungsverfahren & Phytotherapie & Phytotherapie & Homöopathie & Homöopathie \\
\hline Phytotherapie & Atemtechniken & Hydrotherapie & Phytotherapie & Körpertherapien \\
\hline
\end{tabular}

Anzumerken ist, dass die letztgenannte Untersuchung von Büssing et al. (2011) ältere Studienteilnehmer einschloss. Ältere Patienten in den USA bevorzugen im Vergleich $\mathrm{zu}$ dieser deutschen Untersuchung bei den KM-Mitteln eher Nahrungsmittelzusätze, Heilkräuter und Megavitamine sowie bei den KM-Methoden eher spirituelle Methoden und Chiropraktik (Cheung 2007).

Die oben genannten Methoden werden offenbar im Sinne individueller Präferenzmuster genutzt, also nicht auf der Grundlage kollektiver Überzeugungen, die für die gesamte Nutzergruppe typisch sind. Aufschlussreich ist diesbezüglich die Untersuchung von Swartzman et al. (2002), nach der individuelle Entscheidungen auf fünf Dimensionen getroffen werden (s. Tab. 2).

Tab. 2 Präferenzdimensionen (nach Swartzman et al. 2002)

\begin{tabular}{lll}
\hline Dimension & Extrem 1 (Beispiele) & Extrem 2 (Beispiele) \\
\hline positiv - negativ & schmerzhaft, gefährlich, Nebenwir- & effektiv, der Persönlichkeit entsprechend, \\
& kungen (Chirurgie, Injektionen, & traditionell (Entspannung, physische \\
& Elektrostimulation) & Aktivität, Spiritualität)
\end{tabular}

\footnotetext{
${ }^{1}$ Die Untersuchung wurde mit weiblichen Studierenden durchgeführt $(n=173)$ und soll hier nur einen methodisch exemplarischen Charakter haben. Weitere Dimensionalitätseinschätzungen von Patienten finden sich bei Bishop et al. (2007).
} 


\begin{tabular}{|c|c|c|}
\hline Dimension & Extrem 1 (Beispiele) & Extrem 2 (Beispiele) \\
\hline Behandlungskontrolle & $\begin{array}{l}\text { vom Behandler kontrolliert, zu- } \\
\text { meist teuer (Massage, Akupunktur, } \\
\text { Chiropraktik) }\end{array}$ & $\begin{array}{l}\text { vom Patienten kontrolliert, Erfolg abhän- } \\
\text { gig von dessen Anstrengung (Schmerzun- } \\
\text { terdrückung, Gewichtsreduktion, physi- } \\
\text { sche Aktivität) }\end{array}$ \\
\hline konventionell - alternativ & $\begin{array}{l}\text { gut erforscht, traditionell, effektiv } \\
\text { (Chirurgie, Injektionen, orale } \\
\text { Medikamente) }\end{array}$ & $\begin{array}{l}\text { natürlich, kräftigend, unter Kontrolle des } \\
\text { Patienten (Spiritualität, Entspannung, } \\
\text { Phytopharmaka) }\end{array}$ \\
\hline invasiv - nicht invasiv & invasiv (Chirurgie, Injektionen) & nicht-invasiv (Entspannung, Stretching) \\
\hline $\begin{array}{l}\text { Medikament - } \\
\text { Nicht-Medikament }\end{array}$ & $\begin{array}{l}\text { Nebenwirkungen gefährlich (orale } \\
\text { Medikation mit oder ohne Rezept) }\end{array}$ & $\begin{array}{l}\text { von der Routine abweichend, abhängig } \\
\text { von der Anstrengung des Behandlers oder } \\
\text { des Patienten }\end{array}$ \\
\hline
\end{tabular}

Schließlich ist zu berücksichtigen, dass nur ein kleiner Teil der KM-Nutzer radikal „alternative“ Angebote präferiert und konventionelle Behandlungen grundsätzlich ablehnt. Der weitaus größte Teil der KM-Nutzer bedient sich beider Varianten des Gesundheitssystems, wobei allerdings der behandelnde (konventionelle) Arzt häufig nichts von einer „alternativen“ Parallelbehandlung oder -medikation weiß.

\section{KM-Nutzer-Gruppen}

KM-Nutzer stellen eine durchaus heterogene Gesamtgruppe dar: Unter ihnen gibt neben den Prinzipalisten die von der konventionellen Medizin Enttäuschten, aber auch die Opportunisten, die irgendwelchen Trends folgen, sowie die schon erwähnten Mehrfach-Nutzer, die ihren privaten Medizin-Pluralismus pflegen (vgl. Bishop et al. 2007).

Der KM-Gebrauch bei Gesunden scheint auch zu korrelieren mit einem gesundheitsbewussten und präventionsorientierten Lebensstil (Sporttreiben, geringer Alkoholkonsum u.ä.), geringer Inanspruchnahme eines Hausarztes und Zurückhaltung gegenüber verschreibungspflichtigen Medikamenten. Sie sind stärker interessiert, Gesundheitsentscheidungen für sich selbst zu treffen als Nicht-KMNutzer bzw. bereits erkrankte Personen (vgl. Frass et al. 2012).

Eine Umfrage des Allensbach-Instituts (2006) zeigte, dass im Erkrankungsfall mehr als die Hälfte der Bundesbürger sowohl mit Naturheilmitteln und Komplementärmedizin als auch mit Schulmedizin behandelt werden wollen. Etwa ein Drittel präferierte eine rein schulmedizinische Therapie. Weniger als 10\% wollten ausschließlich KM benutzen (Dobos et al. 2006). 


\section{Typische Indikationen für die KM-Nutzung}

In Deutschland benutzen rund 60\% der erwachsenen Bevölkerung mehr oder weniger häufig KM-Methoden und -Produkte zur Behandlung leichter, aber auch chronifizierter Erkrankungen, daneben zur Prävention sowie zur Linderung von Nebenwirkungen durch konventionelle Medikamente und Methoden.

- Die Hauptindikationen in der KM sind nach einer deutschen Untersuchung (Härtel \& Volger 2004) Rückenschmerzen (57,2\%), Erkältungen (29,0\%), Kopfschmerzen (18,7\%), Abgespanntheit (14,6\%), gastrointestinale Probleme (12,3\%) und Allergien (11,4\%).

- Ähnlich listen Bücker et al. (2008) folgende Indikationen für Deutschland auf: Chronische Schmerzen (45,3\%), Kreislaufstörungen (32,9\%), Erkältungskrankheiten ohne Fieber (27,8\%). In der Untersuchung von Marstedt (2002) wurde die $\mathrm{KM} \mathrm{zu} \mathrm{23 \%} \mathrm{bei} \mathrm{chronischen} \mathrm{Erkrankungen,} \mathrm{zu} \mathrm{30 \%} \mathrm{bei} \mathrm{akuten} \mathrm{Er-}$ krankungen, zu 16\% bei allgemeinen Beschwerden und Befindlichkeitsstörungen und zu 31\% zur Krankheitsvorbeugung genutzt.

Im internationalen Vergleich werden KM-Methoden aber auch häufig bei akuten oder überstandenen Krebserkrankungen benutzt (Molassiotis et al. 2005). Etwa 40\% der Krebs-Patienten bedienen sich solcher Verfahren und Produkte, und dies offenbar aus Überzeugung und nicht, weil sie mit der konventionellen Medizin unzufrieden sind. Umgekehrt verschreiben auch 50\% der Ärzte solche Methoden oder Produkte, - entweder auf Wunsch des Patienten oder weil sie selbst glauben, dass diese helfen könnten (Schlaeppi et al. 2005).

\section{Marktpotenzial}

Bei den Heilberufen (in erster Linie niedergelassene Ärzte und Heilpraktiker) sind offenbar das wirtschaftliche Potenzial einerseits und Angebot sowie Bedarfslage andererseits erkannt worden. So werden in diesem Teil des Gesundheitsmarktes rund 9 Milliarden Euro umgesetzt, wovon die Krankenkassen rund 4 Milliarden Euro hierzulande erstatten. Und knapp 50.00o Ärzte in Deutschland (150.ooo Ärzte in der EU) bieten alternative und komplementäre Heilverfahren zusätzlich an; dazu kommen rund 20.0oo Heilpraktiker sowie Anbieter aus anderen Gesundheitsberufen. Nach dem Stand von 2006 (Joos et al. 2008) führen Ärzte folgende Zusatzbezeichnungen aus dem KM-Bereich: Chiropraktik (17.591), Naturheilverfahren (14.497), physikalische Therapie (6.146), Homöopathie (6.073) und Balneologie/Klimaheilkunde (2.886). Die Zahlen derjenigen Ärzte, die Akupunktur praktizieren, werden auf mindestens 10.000 geschätzt. Dazu kommen jene Ärzte, welche auch ohne Zusatzbezeichnung - mehr oder weniger seriöse - KMAngebote im Rahmen von individuellen Gesundheitsleistungen umsetzen.

Auf dem „Markt“ für KM-Angebote konkurrieren in erster Linie Ärzte und Heilpraktiker. Letztere profitieren vor allem von der Zeitknappheit niedergelassener Ärzte, indem sie vergleichsweise mehr Zeit und Zuwendung bieten und eher die Sprache der Patienten sprechen. Allerdings sind sie, wie Ärzte kritisieren, oft 
nicht genügend diagnostisch und therapeutisch qualifiziert, um umfassend aufklären und negative Wechselwirkungen mit konventionellen Medikamenten ausschließen zu können (Joos et al. 2008). Beide Berufsgruppen grenzen sich wiederum von den Anbietern „esoterischer“ Verfahren ab, die eine durchaus beträchtliche Nachfrage bedienen. Für eine ökonomische Gesamtbetrachtung müssen schließlich der Wellness-Markt (einschließlich „medical wellness“) ebenso wie der Markt für sog. OTCs (Over-The-Counter) zumeist in Form von Nahrungsergänzungsmitteln und andere Heilmittel für die Selbstversorgung mit einbezogen werden. Ärzte können hier zumindest eine beratende Funktion einnehmen, falls Patienten bereit sind oder es wünschen, darüber mit ihrem Arzt zu sprechen.

\section{KM in Krankenhäusern und Kliniken}

Für Krankenhäuser, die entweder schon einem komplementär-alternativen Ansatz verpflichtet sind oder eine Angebotserweiterung in diese Richtung erwägen, sind die oben beschriebenen Motive von KM-Nutzern durchaus von Bedeutung. Es reicht allerdings nicht allein aus, sich auf die entsprechenden Angebote zu konzentrieren, da auch das jeweilige Umfeld (Ausstattung, Umgebung, Qualifikation des Personals) mit dem gewählten Ansatz zumindest verträglich sein muss. Im Folgenden werden Krankenhäuser und Kliniken genannt, die einem KM-Ansatz folgen und deren Merkmale im Sinne eines bench-marking aus den jeweils angegebenen Web-Seiten zu entnehmen sind. Aber auch konventionelle Krankenhäuser bemühen sich zumindest auf Web-Seiten, den Interessen von Patienten und ihren Angehörigen nach einem KM-Zusatzangebot insbesondere in der Onkologie und Pädiatrie entgegenzukommen (vgl. Brauer et al. 2010).

\section{Naturheilkundliche Schwerpunktsetzung}

Die „klassische“ Naturheilkunde mit ihrem Schwerpunkt auf Lebensstilmodifikation, Hydro- und Phytotherapie ist zu unterscheiden von sog. Naturheilverfahren, welche auch erweiterte Verfahren wie z.B: Schröpfen, Manualtherapie, Symbioselenkung, Blutegel u.a.m. mit einschließen. Naturheilkunde setzt auf die „Natur“ als Heilkraft und versucht, den Organismus bei seinen Bestrebungen zum gesunden Gleichgewicht $\mathrm{zu}$ unterstützen. Eine solche Förderung der „Selbstheilungskräfte“ wirkt dann auch gesundheitsstärkend. gesundheitserhaltend und präventiv. Störungen des Kräftegleichgewichts im Organismus auszugleichen und in diesem Sinne auch präventiv zu wirken. „Selbstheilungskraft“ soll durch reizregulatorische, physiologisch optimierende oder manchmal auch schonende Maßnahmen der Naturheilkunde gestärkt werden. Zur „klassischen“ Naturheilkunde im engeren Sinne zählen Ernährungstherapie, Phytotherapie, Hydrotherapie, Bewegungstherapie und Ordnungstherapie. In diesem Rahmen finden u.a. folgende Techniken Anwendung: Medizinische Bäder, Dampf- und Moorbäder, Elektrotherapie, Lichttherapie, Wärme- und Kältetherapie, Güsse, 
Wickel, Wassertreten, Massage, Krankengymnastik, Reflektorische Therapie u.a.m.

\section{Anthroposophische Schwerpunktsetzung}

Die Anthroposophische Medizin geht von der Vorstellung einer Existenz auf vier - körperlichen und seelischen - Ebenen aus, die in ihrer Wechselwirkung gestört sein können. Zu den Methoden der Anthroposophischen Medizin zählen speziell (ähnlich wie in der Homöopathie) Verdünnungsmethoden von mineralischen, pflanzlichen und Metallen (insbesondere Mistelpräparate), aber auch bestimmte Bewegungstherapien und Heilbäder. Diese werden vorwiegend bei Tumoren, Arthrosen und autoimmunologischen Erkrankungen angewendet.

\section{Homöopathische Schwerpunktsetzung}

Die Homöopathie geht davon aus, dass der Körper über Selbstheilungskräfte verfügt, die man durch geeignete Substanzen - wenn auch in stark verdünnter und durch ein spezielles Herstellungsverfahren „potenzierter“ Form - anregen kann. Dabei handelt es sich um Substanzen, die in ihrem Arzneimittelbild dem Krankheitsbild funktional ähnlich sind (Prinzip der Ähnlichkeit). Die Verabreichung erfolgt je nach individueller Konstitution. In der „klassischen“ Homöopathie werden solche Substanzen als Einzelsubstanzen verabreicht, in der Komplexhomöopathie miteinander kombiniert, in der „modernen“ Homöopathie im Sinne der „integrativen Medizin“ zum Teil in Kombination mit klassischen Arzneien verabreicht. Angewendet werden homöopathische Heilmittel bei leichteren Erkrankungen (sog. Bagatellerkrankungen), aber auch vielfach bei Hauterkrankungen, Allergien, gastroenterologischen, immunolgischen und rheumatischen sowie psychosomatischen Erkrankungen, und hierbei insbesondere jeweils bei chronischen Erkrankungen. Wegen der geringen Nebenwirkungen werden solche Heilmittel häufig für Kinder und Jugendliche empfohlen und haben sich auch in der Selbstmedikation stark verbreitet.

\section{TCM-Schwerpunktsetzung}

Der Terminus TCM wird unter Spezialisten kritisch und kontrovers gesehen und häufig wird daher von „chinesischer Medizin“ gesprochen, da die traditionellen Wurzeln des heute etablierten Verfahrensspektrums sowie die theoretischen Grundlagen nicht ganz deckungsgleich sind. In der Regel wird heute unter „Traditioneller Chinesischer Medizin“ (TCM) eine Selektion von teilweise sehr alten körperzentrierten Verfahren verstanden, die auf unterschiedlichen theoretischen Grundlagen beruhen. Am bekanntesten sind das Yin- und Yang-Prinzip sowie die Annahme einer fluiden Energie (Qi), auf die beispielsweise die Akupunktur Bezug nimmt. Neben Reiztechniken wie Akupunktur und Akupressur zählen zur TCM auch Bewegungstherapien (z.B. Qigong), Massage (Tui Na) sowie Diätetik und Phytotherapie. Gemeinsam ist diesen, dass sie das „Qi“ anregen und Blockaden 
beseitigen wollen. Das Anwendungsspektrum der TCM ist sehr breit, wobei sich insbesondere Akupunktur bei verschiedenen Schmerzarten bewährt hat.

\section{Organisationsformen der medizinischen Versorgung}

Die genannten Schwerpunktsetzungen werden in recht unterschiedlichen Organisationsformen realisiert. So kann unterschieden werden zwischen verschiedenen Typen:

- Vollkrankenhaus mit spezifischer komplementärer, naturheilkundlicher oder integrativer Ausrichtung und bevorzugter Anwendung einschlägiger Behandlungsmethoden (z.B. anthroposophische Krankenhaus)

- komplementärmedizinisch ausgerichtete Fachabteilung in einem Krankenhaus (z.B. Naturheilkunde in Verbindung mit Innerer Medizin)

- Fachkrankenhaus/Klinik mit spezialisierter Versorgung (z.B. TCM-Klinik).

- Konsiliar- oder Ambulanzdienste mit einem komplementärmedizinischen Dienst oder mehreren Diensten.

Daneben gibt es Varianten im Rahmen der „integrierten Versorgung“, bei denen - zum Teil über Medizinische Versorgungszentren - einzelne Krankenhausabteilungen fallweise mit spezialisierten KM-Kliniken (z.B. in der Rheumatologie) und mit niedergelassenen Ärzten oder Heilpraktikern zusammenarbeiten.

Aus Patientensicht haben die jeweiligen Organisationsformen gewisse Vor- und Nachteile:

- Eher weltanschaulich homogen ausgerichtete Krankenhäuser, wie z.B. die anthroposophisch erweiterten Häuser bieten den Vorteil einer in diesem Rahmen konsequenten Behandlung und einer auf gemeinsame Ziele und Methoden festgelegten Mitarbeiterschaft sowie eines kompletten integrativen Settings; bei bestimmten Krankheiten sind solche Häuser möglicherweise einseitig bzw. überfordert.

- Fachabteilungen mit KM-Ausrichtung in großen Krankenhäusern haben den Vorteil, dass jederzeit eine Kooperation mit „konventionell“ arbeitenden Abteilungen möglich und im Falle von Komorbidität auch eine arbeitsteilige Behandlung organisatorisch $\mathrm{zu}$ bewältigen ist; Nachteile dürften in der mangelnden Anerkennung durch das organisatorische Umfeld liegen.

- Spezialisierte Kliniken bieten ähnliche Vorteile wie die o.e. weltanschaulich ausgerichteten Krankenhäuser, können aber aufgrund ihre geringeren Größe mehr Geborgenheit vermitteln und ein individualisierteres Vorgehen ermöglichen; Nachteile liegen vor allem in dem eingeschränkten Indikations- und Therapiespektrum. 


\section{Leitgedanken zur Implementierung von KM in Krankenhäuser der Regelversorgung}

Bei der Implementierung von KM-Angeboten in die Regelversorgung kann man auf einige bisherige Erfahrungen von Krankenhäusern/Kliniken Bezug nehmen, die zumindest einzelne Fachabteilungen oder Stationen diesbezüglich neu gestaltet haben.

Die erfolgreiche Praktizierung von KM-Methoden setzt eine positive Einstellung der beteiligten Behandler (Ärzte, Pflegekräfte) voraus. Diese liegt bezüglich bestimmter Methoden zumindest bei niedergelassenen Ärzten in Deutschland überwiegend vor: Am meisten geschätzt werden - in absteigender Reihenfolge - physikalische Therapie, Phytotherapie, Bewegungstherapie, Ernährungstherapie, Massage, Entspannungstechniken, Homöopathie, Akupunktur, sonstige TCM (Stange et al. 2008). Eine überwiegend positive Einschätzung wird auch aus anderen Ländern berichtet. (z.B. Milden 2004), wobei aber je nach Fachrichtung unterschiedliche KM-Methoden für nützlich gehalten werden. Manche Ärzte glauben auch nur, dass sich durch die Nutzung solcher Methoden die Patientenzufriedenheit und die Attraktivität des Hauses erhöhen lässt (Wahner-Roedler et al. 2006).

- Auch konventionell orientierte Ärzte müssen mit ihren Patienten offen über KM-Themen sprechen können und sollten nicht die Vorgehensweise der integrativen Medizin offen der unbewusst konterkarieren. Dies geschieht in der Onkologie (Oh et al. 2010), Neurochirurgie (Wu et al. 2009) oder Pädiatrie (Cuzzolin et al. 2003, Roth et al. 2009) noch unzureichend. Hindernisse sind nicht nur mangelnde Zeit, sondern auch das zu geringe Vorwissen und die Bereitschaft, sich auf zum Teil befremdlich anmutende Krankheitstheorien einzulassen.

- Die Bedeutung und Wirksamkeit einer KM-orientierten Abteilung im Krankenhaus oder einzelner Spezialisten hängt sehr davon ab, welchen Status und welche Ausstattung man ihnen zubilligt. Die Beschäftigung auf der Basis von Teilzeit- und Fristverträgen schafft nicht die Voraussetzungen für eine kontinuierliche Betreuung von Patienten, Kommunikation mit anderen Professionellen oder Evaluation (Soklaridis et al. 2009). Dabei ist zu berücksichtigen, dass verschiedene KM-Methoden auch einen unterschiedlich hohen zeitlichen Einsatz erfordern; so braucht man beispielsweise für Akupunktur deutlich weniger Aufwand als für eine umfassende anthroposophische Behandlung (Widmer 2006).

- Die Integration kann nicht gelingen ohne Information und Entscheidungsmöglichkeiten für Patienten. Dazu kann auf bisher erkannte Präferenzmuster und Merkmale bestimmter Patientengruppen zurückgegriffen werden: Bei onkologischen Patienten sind solche Merkmale z.B. höheres Alter, höhere Schulbildung und weibliches Geschlecht (Schernhammer et al. 2009), in der Rheumatologie z.B. höhere Schulbildung und Vorliegen einer Fibromyalgie (Breuer et al. 2006). So sind beispielsweise Patienten mit psychosomatischen 
Störungen eher für KM-Methoden zu gewinnen als Patienten mit gastroenterologischen oder kardiologischen Problemen (Huber et al. 2004). Ähnliche Profile lassen sich auch für andere Indikationen bestimmen (Hoefert \& Uehleke 2009).

- Der Integrationsprozess von KM in ein bisher eher konventionell orientiertes Krankenhaus bedarf einer sorgfältig geplanten Implementationsstrategie. Wie auch bei anderen Innovationen bedarf es zunächst der Unterstützung durch die Klinikleitung im Sinne eines top-down-Prozesses; andererseits kann auch die Nachfrage durch Patienten im Sinne eines bottom-up-Prozesses hilfreich sein (Clement 2006). Weitere Instrumente sind Arbeitsgruppen aus konventionellen und KM-freundlichen Ärzten, KM-Fortbildung, Anreize zum Experimentieren und begleitende Evaluation (Gamus \& Pintov 2007).

- Bei derartigen Maßnahmen müssen ebenfalls die Pflegekräfte integriert werden, welche bestimmte KM-Methoden bereits als „ihr“ Feld definieren und dort häufig Selbstbestätigung finden (z.B. bei körpernahen Methoden wie Therapeutic Touch oder Reiki). Insbesondere die Methoden der Hydro- und Physiotherapie werden traditionell auch durch geschulte Pflegekräfte appliziert. Allerdings korrespondiert die positive Einstellung von Pflegekräften zur KM nicht immer mit der ausreichenden Kenntnis über Nebenwirkungen, die es auch bei KM-Methoden gibt, und bringt sie damit nicht selten in Gegensatz zu Ärzten (Hann et al. 2004).

Bleibt schließlich die Frage der Finanzierung bzw. Finanzierbarkeit von KMEinrichtungen. Für die stationären und teilstationären Leistungen gelten die gleichen Fallpauschal-Entgeltsysteme wie für konventionelle Einrichtungen. Der personelle Mehraufwand wird in der Regel über zu vereinbarende Zusatzentgelte abgebildet. Im Bereich der ambulanten Leistungen sind zum einen die Selbstzahlungen der Patienten die Basis (GOÄ; IGEL), zum anderen werden zunehmend häufig Selektivverträge $\mathrm{zu}$ einzelnen Methoden oder auch komplementärem Netzwerken vereinbart.

Für den stationären Bereich ist entscheidend die Bereitschaft von Krankenkassen, die Leistungserstattung auch bei KM-Therapien nicht infrage zu stellen und durch MDG-Prüfungen auszuhebeln. Die Krankenkassen, der MDK und Behörden sollten daher von vornherein in den Implementierungsprozess eingebunden werden oder zumindest für eine Modellmaßnahme gewonnen werden. Daneben ist zu erwägen, dass die Erweiterung des therapeutischen Standardangebotes um komplementär-alternative Methoden auch dem Ruf eines „patientenorientierten“ Krankenhauses (vgl. Hoefert \& Härter 2010) dienlich ist, der auch der Inanspruchnahme von Standardleistungen zugutekommt.

\section{Literatur}

Barnes, P.M., Powell-Griner, E., McFann, K., Nahin, R.L. (2004). Complementary and alternative medicine use among adults: United States 2002. Adv Data, 343, 1-19. 
Bishop, F.L., Yardley, L., Lewith, G.T. (2007). A systematic review of beliefs involved in the use of complementary and alternative medicine. J Health Psychol, 12 (6), 851-867.

Brauer, J.A., El Sehamy, A., Metz, J.M., Mao, I.J. (2010). Complementary and alternative medicine and supportive care at leading cancer centers: A systematic analysis of websites. I Alternat Complement Med, 16(2), 183186.

Breuer, G.S., Orbach, H., Ekayam, 0., Berkun, Y., Paran, D., Mates, M., Nesher, G. (2006). Use of complementary and alternative medicine among patients attending rheumatology clinics in Israel. IMAJ, 8, 184-187.

Bücker, B., Groenewold, M., Schoefer, Y., Schäfer, T. (2008). Inanspruchnahme von Alternativverfahren bei 1001 deutschen Erwachsenen: Ergebnisse eines bevölkerungsbezogenen Telephonsurveys. Gesundheitswesen, 70: e29-e36.

Büssing, A., Ostermann, T., Heusser, P., Matthiesen, P.F. (2011). Usage of alternative medical systems, acupuncture, homeopathy and anthroposophic medicine, by older German adults. I Chin Integr Med, 9(8), 847-856.

Clement, I.P., Chen, H.F., Burke, D., Clement, D.G., Zazzali, J.L. (2006). Are consumers reshaping hospitals? Complementary and alöternative medicine in U.S. hospitals, 1999-2003. Health Care Manage Rev, 31 (2), 109118.

Cuzzolin, L., Zaffani, S., Murgia, V., Gangemi, M., Meneghelli, G., Chiamenti, G., Benoni, G. (2003). Patterns and perceptions of complementary/alternative medicine among paediatricians and patients' mothers: a review of the literature. Eur | Pediatr, 162 (12), 820-827.

Deloitte Center for Health Solutions (2010). 2010 Survey of health care consumers in Germany. Behaviors, attitudes and unmet needs. www.deloitte.com.

Dobos, G., Deuse, U., Michalsen, A. (Hrsg.) (2006). Chronische Erkrankungen integrativ. Kandern (Narayana).

Eisenberg, D.M., Davis, R.B., Ettner, S.L., Appel, S., Wilkey, S., van Rompay, M., Kessler, R.C. (1998). Trends in alternative medicine use in the United States, 1990-1997. JAMA, 280 (18), 1569-1575.

Falkenberg, T., Lewith, G., Roberti di Sarsina, P., von Ammon, K., Santos-Rey, K., Hök, I., Frei-Erb, M., Vas, J., Saller, R., Uehleke, B. (2012). Towards a pan-European definition of complementary and alternative medicine - a realistic ambition? Forsch Komplementmed, 19, Suppl. 2, 6-8.

Frass, M., Strassl, R.P., Friehs, H., Müllner, M., Kundi, M., Kayne, A.D. (2012). Use and acceptance of complementary and alternative medicine among the genral population and medical personnel: A systematic review. Ochsner I, 12(1), 45-56.

Gamus, D., Pintov, S. (2007). Integration of complementary and alternative medicine services in the hospital setting in Israel. IMAI, 9, 169-170.

Härtel, U., Volger, E. (2004). Inanspruchnahme und Akzeptanz klassischer Naturheilverfahren und alternativer Heilmethoden in Deutschland - Ergebnisse einer repräsentativen Bevölkerungsstudie. Forsch Komplementaermed, 11, 327-334.

Gesundheitsberichterstattung des Bundes (GBE) (2002). Inanspruchnahme alternativer Methoden in der Medizin. Heft 9. www.rki.de.

Hann, D.M., Baker, F., Denniston, M.M., Winter, K. (2004). Oncology professionals' views of complementary therapies: a survey of physicians, nurses, and social workers. Cancer Control, 11 (6), 404-410.

Hoefert, H.-W. (2010). Patienten zwischen konventioneller und komplementär-alternativer Medizin. In: Hoefert, H.-W., Klotter, C. (Hrsg.). Wandel der Patientenrolle. Göttingen (Hogrefe), 217-234.

Hoefert, H.-W., Uehleke, B. (2009). Komplementäre Heilverfahren im Gesundheitswesen. Bern (Huber).

Hoefert, H.-W., Härter, M. (2010). Patientenorientierung. In: Hoefert, H.-W., Härter, M. (Hrsg.). Patientenorientierung im Krankenhaus. Göttingen (Hogrefe).

Huber, R., Koch, D., Beiser, I., Zschocke, I., Luedtke, R. (2004). Experience and attitudes towards CAM - a survey of internal and psychosomatic patients in a German university hospital. Altern Ther Health Med, 10 (1), 32-36.

Joos, S., Musselmann, B., Miksch, A., Rosemann, T., Szecsenyi, I. (2008). The role of complementary and alternative medicine (CAM) in Germany - a focus goup study of GPs. BMC Health Serv Res, 8: 127.

Jütte, R. (1996). Geschichte der Alternativen Medizin. München (Beck).

Kartte, I., Neumann, K. (2008). Der zweite Gesundheitsmarkt. Roland Berger Strategy Consultants. www.rolandberger.com.

Kiene, H. (2001). Komplementäre Methodenlehre der klinischen Forschung. Berlin (Springer).

Milden, S.P. (2004). Physicians' attitudes and practices regarding complementary and alternative medicine. Behav Med, 30, 73-82.

Molassiotis, A., Fernandez-Ortega, P., Pud, D., Ozden, G., Scott, J.A., Panteli, V., Margulies, A., Browall, M., Magri, M., Selcekerova, S., Madsen, E., Milovics, L., Bruyns, I., Gudmundsdottir, G., Hummerston, S., Ahmad, A., 
Platin N., Kearney, N., Patiraki, E. (2005). Use of complementary and alternative medicine in cancer patients: a European survey. Ann Oncol, 16 (4), 655-663.

National Center for Complemenatry and Alternative Medicine (NCCAM) (2008). What is CAM? http://nccam.nih.gov/health/whatiscam/.

Oh, B., Butow, P., Mullan, B., Clarke, S., Tattersall, M., Boyer, M., Beale, P., Vardy, I., Pavlakis, N., Larke, L. (2010). Patient-doctor communication: use of complementary and alternative medicine by adult patients with cancer. I Soc Integr Oncol, 8 (2), 56-64.

Roth, M., Lin, J., Kim, M., Moody, K. (2009). Pediatric oncologists' views toward the use of complemantary and alternative medicine in children with cancer. I Pediatr Hematol Oncol, 31 (3), 177-182.

Schernhammer, E.S., Haidinger, G., Waldhör, T., Vuluc, C. (2009). Attritudes about the use of complementary and alternative medicine in cancer treatment. I Altern Complement Med, 15 (10), 1115-1120.

Schlaeppi, M.R., Jungi, W.F., Cerny, T. (2005). Komplementärmedizin in der Onkologie - eine Einführung. Schweiz Med Forum, 5, 686-694.

Singh, S., Ernst, E. (2009). Gesund ohne Pillen. München (Hanser).

Soklaridis, S., Kelner, M., Love, R.L., Cassidy, J.D. (2009). Integrative health care in a hospital setting: communication patterns between CAM and biomedical practioners. I Interprof Care, 23 (6), 655-667.

Stange, R., Amhof, R., Moebus, S. (2008). Complementary and alternative medicine: attritudes and patterns of use by German physicians in a national survey. I Altern Complement Med, 14 (10), 1255-1261.

Stiftung Warentest (Hg.) (2006). Die Andere Medizin. Berlin (Stiftung Warentest).

Swartzman, L.C., Harshman, R.A., Burkell, I., Lundy, M.E. (2002). What accounts for the appeal of Complementary/Alternative Medicine, and what makes Complementary/Alternative Medicine "alternative"? Medical Decision Making, 22, 431-450.

Wahner-Roedler, D.L., Vincent, A., Elkin, P.L., Loehrer, L.L., Cha, S.S., Bauer, B.A. (2006). Physicians' attitudes toward complementary and alternative medicine and their knowledge of specific therapies: a survey at an academic medical center. Evid Based Complement Alternat Med, 3 (4), 495-501.

Wapf, V., Busato, A. (2007). Patients' motives for choosing a physician: comparison between conventional and complementary medicine in Swiss primary care. BMC Complement Altern Med, 7: 41.

Widmer, M., Dönges, A., Wapf, V., Busato, A., Herren, S. (2006). The supply of complementary and alternative medicine in Swiss hospitals. Forsch Komplementmed, 13 (6), 356-361.

Wu, C., Weber, W., Kozak, L., Standish, L.J., Ojemann, J.G., Ellenbogen, R.G., Avellino, A.M. (2009). A survey of complementary and alternative medicine (CAM) awareness among neurosurgeons in Washington State. I Altern Complement Med, 15 (5), 551-555. 